\title{
Health Care Facility Network Efficiency and Location Crucial for Ebola Treatment Outcome
}

\author{
Jia Bainga Kangbai ${ }^{1,2^{*}}$, Sulaiman Mandoh ${ }^{3}$ and John L McBrayer ${ }^{4}$ \\ ${ }^{1}$ Center for International Health, Ludwig Maximillians University, Munich, Germany \\ ${ }^{2}$ Department of Environmental Health Sciences, Njala University, Sierra Leone \\ ${ }^{3}$ School of Nursing, Midwifery and Indigenous Health, Faculty of Science, Charles Stuart University, Orange, New South Wales, Australia \\ ${ }^{4}$ Massachusetts School of Public Health, University of Massachusetts, Amherst, USA \\ "Corresponding author: Jia Bainga Kangbai, Center for International Health, Ludwig Maximillians University, Leopoldstrass 7, Munich, Germany, E-mail: \\ Jia.Kangbai@Irz.uni-muenchen.de
}

Received date: December 24, 2018; Accepted date: January 01, 2019; Published date: January 07, 2019

Copyright: @ 2019 Kangbai JB, et al. This is an open-access article distributed under the terms of the Creative Commons Attribution License, which permits unrestricted use, distribution, and reproduction in any medium, provided the original author and source are credited.

\begin{abstract}
Background: The application of network analysis in public health research is gaining popularity with the belief that health problems are a product of complex social, political and economic causes. Organisational networks can be used in public health to design and evaluate public health programs. There have been multiple studies in the past relating to public health organisations and HIVIAIDS using organisational networking method. Here we use social network analysis to analyse the association between the network positions of health care facilities and the treatment outcome of Ebola patients from these health care facilities in Sierra Leone.

Methods: We extracted and later analysed treatment outcome data obtained from the Case Report Forms (CRF) of 117 Ebola patients who sought Ebola treatment at the Kenema Government Hospital from June 2014 to April 2015. For ego network purpose, we considered each Ebola referral health care facility as an ego. Poisson regression analysis was used to determine the association between Ebola mortality rates in a health care facility and the ego's structural holes.

Results: The median age, length of stay during Ebola treatment, and the period it takes an Ebola patient to survive treatment following from onset of signs and symptoms were 20 (IQR=17.5 years), 15 (IQR=11 days) and 7 (IQR=5.3 days) respectively. There were differences in ego, network efficiency, effective size and constraint for the factors associated with mortality rates for Ebola patients treated at the Kenema Government Hospital.

Conclusion: Establishing non-redundant contacts within a health care facility network and expanding their locations can reduce Ebola mortality rates during outbreak.
\end{abstract}

Keywords: Ebola patients; HIV/AIDS; Communicable diseases

\section{Introduction}

The application of network analysis in public health research is gaining popularity with the belief that health problems are a product of complex social, political and economic causes. Many network analysis methods can be applied to address public health issues. Network analysis which deals describe, analysis, explores and assesses the structural and relational issues of people or organizations [1]. Networks analysis thus offered a paradigm shift of how to frame and answer public health problems based on the inherent qualities of relational data. Public health network analysis falls into three categories; transmission, social and organizational networks [1]. Transmission network which structures the flow of tangible elements is divided into disease and information transmission networks [1]. Many human communicable diseases are transmitted through the disease transmission network which is vital in determining disease dynamics in a human population [2]. There is paucity of research on information transmission network. One basic tenet of health communication and education is to devise an efficient and educative means of transmitting health information to practitioners, communities, and consumers which can lead to disease risk reduction and health promotion [1]. Currently health communication and education is making use of network analysis method. One seminal network analysis study on health information diffusion across communities examined the diffusion of information about new drugs through informal physician social network [3]. Some studies have shown that network position is associated with family planning knowledge, attitude and possible use $[4,5]$.

Social network focuses less on transmission of tangible element but more on social structure and relationships [1]. Public health experts of social networks have been curious to know how different aspects of social support influenced mentality and physical and behaviour [6,7]. With the advent of social network analysis, access to health resources thus became an important issue in public health. Several studies have associated large social networks with improved health and reduced mortality [8]. Generally, social support and capital are deemed as important players in population health and well-being.

Organisational networks have been used in public health to design and evaluate public health programs. There have been multiple studies in the past relating to public health organisations and HIV/AIDS using 
organisational networking method [9-11]. Several researches have shown that organisations working on the same issue or receiving funding predicts partnering $[12,13]$. Some researchers have identified barriers to inter-organisational relationship such as building connections claiming it limits autonomy [12-14]. The emergence of organisational network analysis has provided insight into the structure and relationships that exists within a public health system and how they affect their performance and outcomes. Ron Burt in 1992 pushed forward the egocentrically-based concept of structural holes which highlights among others the importance of non-redundant relationship between two contacts within a network [15]. Structural hole is a collection of measures that includes ego network efficiency, effectiveness size and constraint [15]. According to Burt, an individual or organisation's opportunities are affected by its relationship with its primary contacts as well as the relationship to those connected to its primary contacts.

In this organisational network analysis, we used graphic, organisational network and statistical analysis to determine the factors that are associated with the number of Ebola patients who died per a referring health care facility when undergoing treatment at the Kenema Government Hospital (KGH).

\section{Methods}

\section{Network data}

We extracted and later analysed treatment outcome data obtained from the Case Report Forms (CRF) of 117 Ebola patients who sought Ebola treatment at KGH from June 2014 to April 2015. The 34 Military Hospital and KGH were among the key Ebola Treatment Centers (ETCs) in Sierra Leone during the 2014-2015 West Africa Ebola outbreak. Dataset analysed in this study can be accessed only with reasonable request to the corresponding author. All Ebola patients whose treatment outcome data were used in this study were confirmed as Ebola positive using quantitative Reverse-TranscriptasePolymerase-Chain-Reaction (RT-PCR) assay with specific EVD primers. Each Ebola patient CRF contains patient demographic information, date of Ebola symptom onset, residential district, treatment outcome, date of admission, date of discharge, and the health care facility the patient was referred from. Ebola patients were referred for treatment to $\mathrm{KGH}$ from the following government and private hospitals; Makeni Government Hospital (MAKGH), Port Loko Government Hospital (PLTCGH), Kenema Government Hospital (KENGH), Koindu Government Hospital (KOINGH), Pujehun Government Hospital (PUJGH), Jui Hospital (JUIHC), Moyamba Government Hospital (MOYGH) and Connaught Government Hospital (CONGH). Ebola patients were also referred for Ebola treatment to $\mathrm{KGH}$ from the following Community Health Centers (CHC); Red Cross CHC (REDCHC), Komende CHC (KOMHC), Lion Heart CHC (LHCHC), Mabeseneh CHC (MABHC), Maloli Junction CHC (MALHC), McCauley CHC (MCLHC), Regional CHC (REGHC), Newton CHC (NEWHC), Petifu CHC (PETHC), and Rokupar CHC (ROKHC).

\section{Ego network}

For ego network purpose, we considered each health care referral facility from which an Ebola patient was referred from to the KGH for treatment as an ego while the other health care facilities to which the ego is connected as alters. We hypothesized that a successful Ebola treatment outcome for patients referred from one health care facility to another depends on the availability or not of other health care facilities (alters) within the health care facility network to which the referring health care facility is connected, as well as the influence the ego has within the health care network.

We first created a relational binary database to indicate whether health care facilities are related or not by coding their relationship with each other in a Microsoft Excel spreadsheet database. Health care facilities relationship were coded 1 if they perform identical health care services or and are located within the same district. Health care facilities relationship were coded 0 if they do not perform identical health care services or and are located within the same district. To visualise the relational binary data we used UCINET 6.624 (Software for Social Network Analysis. Harvard: Analytic Technologies) to construct a relationship matrix and then used NetDraw 2.160 (NetDraw: Graph Visualization Software. Harvard: Analytic Technologies) to visualize all the linkages (coded relationship) connecting each node (health care facility) to produce a network. We later formatted the network by colouring all government and private hospitals green and all community health clinics red.

\section{Structural holes computation}

UCINET was then used to compute the structural holes for the egos that referred Ebola patients to $\mathrm{KGH}$ in order to determine whether a health care facility position within its neighbourhood is associated with the KGH Ebola mortality rates during treatment. Specifically, structural holes were computed in order to determine the association between an egos effect size (Effisize), efficiency (Efficienc), constrains (Constrain), and the number of EVD patients who died from each health care facility during treatment at $\mathrm{KGH}$.

\section{Ebola mortality rate}

Poisson regression analysis was used to determine the association between the number of EVD deaths from an ego at KGH, and the ego's structural holes, district of residence and level of health care services offered by the ego. We hypothesized that the decision to refer an Ebola patient from one health care facility to $\mathrm{KGH}$ is influenced by several factors including access to $\mathrm{KGH}$, information relating to Ebola treatment option at $\mathrm{KGH}$, and the timing of the availability of the information relating to Ebola treatment outcome.

\section{Statistical analysis}

$\mathrm{R}$ software package version 2.15.1 was used for both descriptive and analytical statistics in this study; the source codes are available upon request [16]. A p $<0.05$ was considered as statistical significance for all two-sided statistical tests. Summary statistics (count and proportion) was done to determine the total and proportions of treatment outcome for Ebola patients referred from each health care facility to receive treatment in $\mathrm{KGH}[17,18]$.

\section{Ethics and privacy}

The Institutional Review Board at Njala University in Sierra Leone approved this study and provided ethical clearance. The Njala University Institutional Review Board waived the requirement to obtain informed consent on the grounds that the study involves minimal health risk on participating study participants. 
Citation: Kangbai JB, Mandoh S, Brayer JLM (2019) Health Care Facility Network Efficiency and Location Crucial for Ebola Treatment Outcome .

Page 3 of 6

\section{Results}

We analysed the medical records of one hundred and seventeen (117) confirmed Ebola patients who were referred by eighteen health care facilities in Sierra Leone for Ebola treatment to the KGH between the periods August 2014 to November 2014. The ages, length of stay during Ebola treatment (Los), and the period it took an Ebola patient to survive Ebola treatment following onset of signs and symptoms (survival) in this study were not normally distributed (Figure 1).

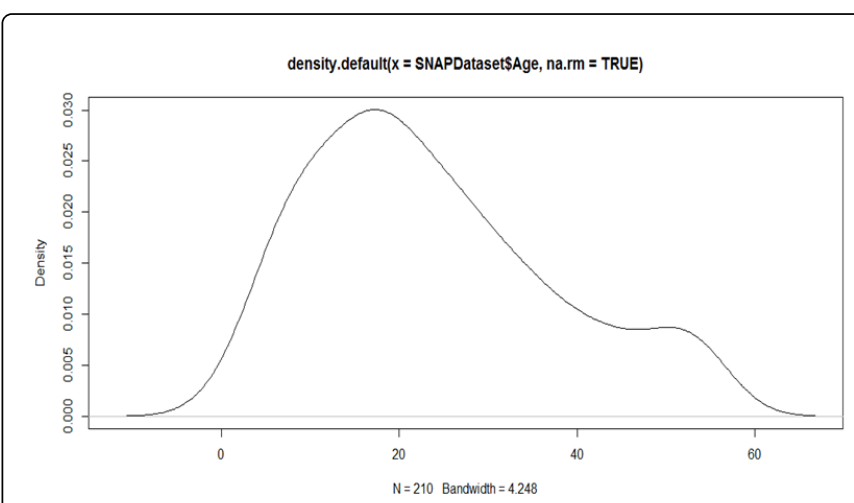

Figure 1: Age distribution of Ebola patients treated at the Kenema Government Hospital.

From Figure 1 the age distribution of Ebola patients who received treatment at the Kenema Government Hospital from August 2014 to November 2014 was not randomly distributed.

The median age, length of stay during Ebola treatment (Los) and the period it takes an Ebola patient to survive treatment following onset of signs and symptoms (survival) of EVD patients were 20 (IQR=17.5 yrs), 15 (IQR=11 days) and 7 (IQR=5.3 days) respectively.

Majority (56\%) of the EVD patients was females and was cured and released alive (59\%) following treatment. Connaught Government Hospital referred the highest number $(30 \%)$ of Ebola patients for treatment at KGH (Table 1).

\begin{tabular}{|l|l|}
\hline Characteristic $\mathbf{n}(\%)$ & $\begin{array}{l}\text { Characteristic } \\
(\%)\end{array}$ \\
\hline Sex & $66(56)$ \\
\hline Male & $51(44)$ \\
\hline Female & \\
\hline Treatment outcome & $69(59)$ \\
\hline Cured & $48(41)$ \\
\hline Dead & \multicolumn{2}{|l|}{} \\
\hline Ebola patients referred from health facility & $35(30)$ \\
\hline Connaught Government Hospital & $8(7.0)$ \\
\hline Jui Chinese Hospital & $29(25)$ \\
\hline Kenema Government Hospital & $2(2.0)$ \\
\hline Koindu Government Hospital & $12(10)$ \\
\hline Port Loko Government Hospital & \\
\hline
\end{tabular}

\begin{tabular}{|l|l|}
\hline Moyamba Government Hospital & $11(9.0)$ \\
\hline Makeni Government Hospital & $5(4.0)$ \\
\hline Pujehun Government Hospital & $1(0.9)$ \\
\hline Newton Community Health Center & $1(0.9)$ \\
\hline Mabeseneh Community Health Center & $1(0.9)$ \\
\hline Red Cross Community Health Center & $2(2.0)$ \\
\hline Komende Community Health Center & $1(0.9)$ \\
\hline McCauley Community Health Center & $1(0.9)$ \\
\hline Petifu Community Health Center & $1(0.9)$ \\
\hline Lion Heart Community Health Center & $3(3.0)$ \\
\hline Maloli Community Health Center & $1(0.9)$ \\
\hline Regional Community Health Center & $1(0.9)$ \\
\hline
\end{tabular}

Table 1: Distribution of the characteristics of Ebola patients and treatment mortality.

More EVD cases were referred from Connaught Government Hospital than from any other healthcare facilities in the country.

We used Net Draw to visualize the relational binary data depicting the relationship between the various health care facilities that referred Ebola patients for treatment to KGH (Figure 2).

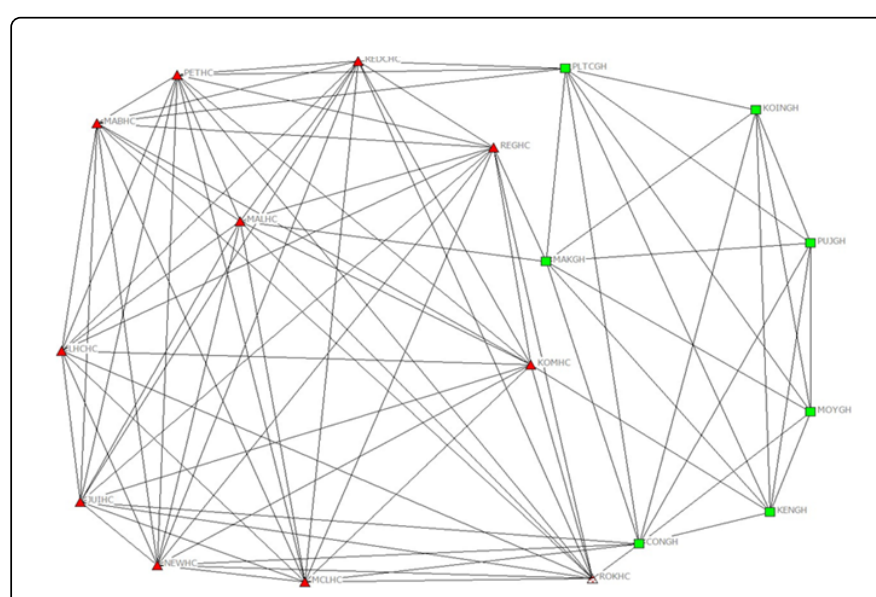

Figure 2: Network displaying the relationship between the various health care facilities that referred Ebola patients for treatment to KGH

UCINET was used to determine the positional advantage of each health care facility within the network. Specifically we determined the structural holes (effective size, efficiency, density and constrains) for each health care facility (ego) that referred Ebola patient to KGH for treatment (Table 2).

\begin{tabular}{|l|l|l|l|l|}
\hline Health care facility & Effisize & Efficiency & Constrain & Mortality (\%) \\
\hline CONGH & 5.800 & 0.580 & 0.324 & 64.0 \\
\hline JUIHC & 2.273 & 0.207 & 0.329 & 12.5 \\
\hline
\end{tabular}


Page 4 of 6

\begin{tabular}{|l|l|l|l|l|}
\hline KENGH & 2.714 & 0.388 & 0.43 & 48.0 \\
\hline KOINGH & 1.000 & 0.167 & 0.560 & 100 \\
\hline KOMHC & 2.818 & 0.256 & 0.307 & 100 \\
\hline LHCHC & 1.000 & 0.100 & 0.361 & 66.0 \\
\hline MABHC & 2.455 & 0.223 & 0.327 & 0.0 \\
\hline MAKGH & 4.000 & 0.500 & 0.385 & 0.0 \\
\hline MALHC & 2.636 & 0.240 & 0.323 & 0.0 \\
\hline MCLHC & 2.273 & 0.207 & 0.329 & 100 \\
\hline MOYGH & 1.000 & 0.167 & 0.560 & 36.0 \\
\hline NEWHC & 2.273 & 0.207 & 0.329 & 100 \\
\hline PETHC & 2.455 & 0.223 & 0.327 & 0.0 \\
\hline PLTCGH & 5.000 & 0.556 & 0.352 & 8.0 \\
\hline PUJGH & 1.000 & 0.167 & 0.560 & 100 \\
\hline REDCHC & 2.455 & 0.223 & 0.327 & 0.0 \\
\hline REGHC & 2.636 & 0.240 & 0.323 & 0.0 \\
\hline ROKHC & 2.273 & 0.207 & 0.329 & 50.0 \\
\hline
\end{tabular}

$\mathrm{p}<0.02)$.There were occupational and district differences in Ebola treatment outcome per referring health care facility.

\begin{tabular}{|l|l|l|l|}
\hline Variables & AOR & $95 \%$ CI & P-value \\
\hline Male & 8.17 & $2.71-2.44$ & 0.72 \\
\hline Age & 1.01 & $9.69-1.05$ & 0.67 \\
\hline District_coded2 & 2.28 & $4.71-1.31$ & 0.32 \\
\hline District_coded3 & 1.32 & $1.69-5.80$ & 0.32 \\
\hline District_coded4 & 3.95 & $2.05-8.16$ & 0.53 \\
\hline District_coded5 & 3.35 & $1.32-6.09$ & 0.70 \\
\hline District_coded6 & 7.62 & $1.26-6.80$ & $0.04 *$ \\
\hline District_coded7 & 9.81 & $3.99-3.78$ & 0.99 \\
\hline District_coded8 & 1.18 & $<0.00-4.94$ & 1.00 \\
\hline District_coded9 & 2.77 & $<0.00-2.00$ & 1.00 \\
\hline Occupation_coded1 & 5.16 & $8.75-3.51$ & 0.08 \\
\hline Occupation_coded2 & 2.53 & $2.35-7.03$ & $0.02 *$ \\
\hline Occupation_coded3 & 4.70 & $3.37-1.07$ & 0.30 \\
\hline Occupation_coded4 & 4.48 & $2.01-2.09$ & 1.00 \\
\hline Occupation_coded5 & 1.51 & $2.91-8.61$ & 0.63 \\
\hline Occupation_coded6 & 2.43 & $5.19-1.25$ & 0.27 \\
\hline Effisize & 8.04 & $8.78-1.61$ & 0.89 \\
\hline Efficiency & 7.51 & $1.73-3.54$ & 0.98 \\
\hline Constrains & 2.33 & $1.29-6.50$ & 0.83 \\
\hline
\end{tabular}

Table 3: Multivariate analysis of factors associated with Ebola treatment outcome for patients treated at KGH from various health care facilities Government Hospital and Pujehun Government recorded the highest (0.56 each) ego constrains while Komende community health centre recorded the lowest $(0.307)$ ego constrain.

Koindu Government Hospital, Pujehun Government Hospital, Newton community health centre, Komende community health centre and McCauley community health centre recorded the highest $(100 \%)$ mortality for Ebola patients treated at KGH. Red Cross community health centre, Petifu community health centre, Maloli community health centre, Regional community health centre, Mabeseneh Community Health Centre recorded no death during Ebola treatment at KGH recorded no mortality for Ebola patients treated at KGH.

\section{Treatment outcome}

Multivariate logistic regression was used to determine the factors that are associated with the odds of being cured during Ebola treatment at $\mathrm{KGH}$ and the results are presented in Table 3. Holding other factors in the model constant, the Adjusted Odd Ratio (AOR) for Ebola patients referred for Ebola treatment from district 6 (Port Loko District) being cured at KGH is increased by 7.62 compared to Ebola patients referred from other districts $(95 \% \mathrm{CI}=1.26-6.80, \mathrm{p}<0.04)$. Also adjusting for other factors in the model, the AOR of an Ebola patient who is a student being cured is 2.53 times greater than for non-student or Ebola patients of other occupation (95\% CI=2.35-7.03,

\section{Factors associated with EVD mortality}

We used Poisson regression analysis to determine the factors that are associated with the mortality ratefor Ebola patients from each health care facility that referred patients to KGH for treatment and the results are presented in Table 3 . There were differences in health care facility, its (ego) network efficiency, effective size and constraint for the factors associated with the mortality ratefor Ebola patients treated at $\mathrm{KGH}$. Adjusting for other covariates in the model, for a unit change in the health care facility the mortality ratefor Ebola patients from each health care facility that refereed patients to $\mathrm{KGH}$ for treatment decreased by 0.77 ( $95 \% \mathrm{CI}=0.75-0.80, \mathrm{p}<0.0005)$. Specifically holding other covariates in the model constant, for a unit change in the ego network efficiency the mortality ratefor Ebola patients from each health care facility that refereed patients to KGH for treatment increased by 1326 (95\% CI=215.84-8444.05, p<0.0005). Adjusting for other covariates in the model, for a unit change in the ego network effective size the mortality ratefor Ebola patients from each health care facility that refereed patients to $\mathrm{KGH}$ for treatment decreased by 0.62 (95\% CI=0.51-0.74, $\mathrm{p}<0.0005)$. Also, adjusting for other covariates in the model, for a unit change in the ego network constraint the mortality ratefor Ebola patients from each health care facility that 
refereed patients to $\mathrm{KGH}$ for treatment increased by $18.85(95 \%$ $\mathrm{CI}=3.86-92.71, \mathrm{p}<0.0005)$.

\section{Discussion}

The Western Area (Freetown) in Sierra Leone referred the highest (35\%) of Ebola cases treated at KGH compared to other areas in the country due to the fact that the Western Area is the most populated region in the country. The Western Area is home to all ethnic groups in Sierra Leone has a population of about 1.4 million [19]. The poor environmental sanitation and abysmal living conditions in the Western Area makes the spread of Ebola easy within its urban population. Ebola is a space-related infection due to the fact that the infection easily spread when people come in contact with infected bodily fluids [20].

There were occupational and district differences in Ebola treatment outcome among Ebola patients referred to $\mathrm{KGH}$ for treatment. Adjusting for other factors in the model, Ebola patients from District 6 (Port Loko District) have an increased AOR of 7.62 of being cured at KGH compared to Ebola patients referred from other districts $(95 \%$ $\mathrm{CI}=1.26-6.80, \mathrm{p}<0.04)$. Also adjusting for other factors in the model, the AOR of a student Ebola patient being cured is 2.53 times greater than for non-student Ebola patient or Ebola patients of other occupation (95\% CI=2.35-7.03, p<0.02).

The increased odds for Ebola patients from District 6 (Port Loko District) being cured compared to Ebola patients from other districts is related to the fact majority (82\%) of patients treated at KGH from District 6 are $<40$ years. Ebola patients who are $>40$ years tend to have unfavourable Ebola treatment outcome compared to those Ebola patients $<40$ years [21]. The increased odds for a student Ebola patient being cured compared to non-student Ebola patient or Ebola patient of other occupation is due to the fact that students have advanced knowledge in identifying the signs and symptoms of Ebola during the Ebola outbreak in Sierra Leone and hence were very much conversant with them. Early identification of the signs and symptoms of Ebola implies early access to treatment and treatment options and hence increasing the odds for favourable treatment outcome. Also, because student Ebola patients were $<40$ years they have increased odds for a favourable treatment outcome [21].

There were health care facility, health care facility (ego) network efficiency, effective size and constrain differences in the mortality rates of Ebola patients from other health care facilities that were treated at $\mathrm{KGH}$. Holding other factors in the model constant, health care facility (IRR $=0.77,95 \%$ CI $0.75-0.80, \mathrm{p}<0.0005)$, and health care facility network effective size (IRR=0.62, 95\% CI 0.51-074, $\mathrm{p}<0.0005$ ) were associated with decreased mortality rate of Ebola patients from other health care facilities who were treated at KGH. In addition to the age of an Ebola patient, Ebola treatment outcome is also associated with the type of treatment being offered at the Ebola holding center of the health care facility where a suspected Ebola patient is first monitored prior to referral for treatment. Health care facilities which provides suspected Ebola patients with fluid replacement therapies and immune boost medications prior to treatment offers patient greater odds for a successful treatment outcome. Fluid replacement therapies and immune boost medications has been associated with positive Ebola treatment outcome [22-24]. In Sierra Leone most of the Ebola holding centers outside the cities did not provide fluid replacement therapies or immune boost medications during the West Africa Ebola outbreak in 2014-2015.
The decreased mortality rate of Ebola patients from health care facilities who were treated at KGH due to a unit change in health care facility network effective size is related to the fact that a change in the health care facility network effective size will results into an increase in the number of health care facilities within the network which implies more competition for the available Ebola treatment facilities and treatment options. During disease outbreak such as the Ebola outbreak in Sierra Leone in 2014-2015 an increase in the number of health care facilities within the network will surge up the number of Ebola patient death for that health care facility in that network due to reduction as a result of competition for the necessary benefit required for Ebola treatment.

There were health care facility (ego) network efficiency and constrain differences in the mortality rate of Ebola patients from other health care facilities who were treated at KGH. Holding other factors in the model constant, health care network efficiency (IRR=1326, 95\% $\mathrm{CI}=215.84-8444.05, \quad \mathrm{p}<0.0005), \quad$ and constrain $\quad(\mathrm{IRR}=18.85,95 \%$ $\mathrm{CI}=3.86-92.71, \mathrm{p}<0.0005)$ were associated with increased mortality rate of Ebola patients from other health care facilities who were treated at $\mathrm{KGH}$.

The increased mortality rate of Ebola patients from health care facilities who were treated at KGH due to a unit change in health care facility network efficiency is related to the fact that a change in the health care facility network efficiency means a reduction in how much benefit a health care facility gets per each connection within its network. High health care facility network efficiency implies that the health care facility that referred the Ebola patient has many nonredundant secondary contacts per primary contacts (i.e. a high yield per primary contact). In this context because non-redundant contacts are not connected to the ego health care facility which referred these Ebola patients to KGH this implies that most of the benefits to be enjoyed by the ego health care facility will be lost because of the strong connections between the primary contacts and the non-redundant contacts. This strong connection could lead to the primary and nonredundant contacts to connive to prevent the flow of sensitive and crucial Ebola treatment information and treatment options to the ego health care facility. This lack of benefit for the ego health care facility here implies delays or no referral for Ebola treatment, the lack of access to Ebola treatment options information which can lead to inappropriate timing to access Ebola treatment options and treatment centers information that is circulating within the health care facility network. Additionally, in a disease outbreak situation like the 2014-2015 West Africa Ebola outbreak in Sierra Leone were health care facilities in the country were referring Ebola patients for treatment to various Ebola treatment centers in the country these non-redundant health care facilities and their primary contacts can establish high structural equivalence. Structural equivalence measures redundancy between primary and secondary contacts and can be used to demonstrate that contacts occupying or performing similar position or functions within a network will link alters or actors of the same third party contacts. Such structural equivalence can lead to a stronger relationship between the non-redundant and the primary contacts of an ego which can also lead to a fast processing and early release of crucial Ebola treatment information for these non-redundant and their primary contacts. The quick and early release of crucial Ebola treatment information by health care facilities within a network with high number of non-redundant contacts, could lead to early referrals for both non-redundant and primary contacts but not for the ego health care facility. 
Also, in a typical disease outbreak situation like the 2014-2015 West Africa Ebola outbreak in Sierra Leone where treatment options were limited and the number of patients surmounted, non-redundant contacts within a health care facility network can become screeners due to the high competition to access Ebola treatment information and options. In such a situation these non-redundant contacts within the health care facility network tends to dictate which health care facility should have access to a particular treatment centers and options. Additionally, non-redundant contacts with crucial Ebola treatment information within the network can communicate through their primary contacts with other health care facilities where similar useful Ebola treatment information and options are likely to be generated or disseminated which can be communicated among themselves but not shared with the ego health care facility.

The increase in the mortality rate of Ebola patients for health care facility who were treated at KGH due to a unit change in the health care facility network constraint is related to the fact that a change in a health care facility network constraint leads to a decrease in the number of opportunities that an ego health care facility is receiving from its primary contacts due to the primary contacts connections with other (secondary) contacts the ego is trying to reach. Network constraint is a structural hole measure that determines the extent an ego's connections are to others which are connected to one another. In this context a high ego health care facility network constraint means that ego health care facility's primary contacts all have other contacts which that ego health care facility is trying to be in contact with. And because of such high level of connections between the primary contacts and the secondary contacts to which the ego health care facility wants to connect with, the ego health care facility will be highly constrained since it will lose its freedom to take action on many health related issues including referring its patients for Ebola treatment in other health care facilities. This is expected especially if the ego health care network had a long standing relationship and have invested a significant portion of its resources (time etc.) in its primary connections which also has a strong relationship with the secondary contacts that the ego health care facility is trying to reach. With such high ego health care facility network constraint critical decisions such as patient referral and treatment option to be implemented will be slow to be arrived at which can lead to increase in the mortality rate of Ebola patient from that health care facility.

\section{Conclusion}

We were able to demonstrate that the diversity of a health care facility is more important for reducing Ebola mortality during an outbreak period than the number of health care facilities it is connected to. Establishing non-redundant contacts within a health care facility network and expand their locations can reduce Ebola mortality during treatment.

\section{References}

1. Luke DA, Harris JK (2007) Network analysis in public health: History, methods, and applications. Annu Rev Public Health,28: 69-93.

2. Eames KTD, Keeling MJ (2002) Modeling dynamic and network heterogeneities in the spread of sexually transmitted diseases. Proc Natl Acad Sci 99:13330-13335.
3. Coleman J, Katz E, Menzel H (1967) Medical innovation: A diffusion study. Indianapolis. IN: Bobbs-Merrill 46: 291.

4. Boulay M, Storey JD, Sood S (2002) Indirect exposure to a family planning mass media campaign in Nepal. J Health Commun 7: 379-399.

5. Valente TW, Unger J, Johnson AC (2005) Do popular students smoke? The association between popularity and smoking among middle school students. J Adolesc Health 37: 323-329.

6. Berkman LF (1984) Assessing the physical health effects of social networks and social support. Annu Rev Public Health 5: 413-432.

7. Kessler RC, Price RH, Wortman CB (1985) Social factors in psychopathology: stress, social support, and coping processes. Annu Rev Psychol 36: 531-572.

8. House JS, Robbins C, Metzner HL (1982) The association of social relationships and activities with mortality: prospective evidence from the Tecumseh Community Helath Study. Am J Epidemiol 116: 123-140.

9. Kwait J, Valente TW, Celentano DD (2001) Interorganizational relationships among HIV/AIDS service organizations in Baltimore: a network analysis. J Urban Health 78: 468-487.

10. Shumate M, Fulk J, Monge P (2005) Predictors of the International HIVAIDS INGO Network over time. Hum Commun Res 31: 482-510

11. Wright ER, Shuff IM (1995) Specifying the integration of mental health and primary health care services for persons with HIV/AIDS: The Indiana integration of care project. Soc Netw 17: 319-340.

12. Bolland JM, Wilson JV (1994) Three faces of integrative coordination: a model of interorganizational relations in community-based health and human services. Health Serv Res 29: 341-366.

13. Wickizer TM, Von Korff M, Cheadle A, Maeser J, Wagner EH, et al. (1993) Activating communities for health promotion: a process evaluation method. Am J Public Health 83: 561-567.

14. McKinney MM, Morrissey JP, Kaluzny AD (1993) Interorganizational exchanges as performance markers in a community cancer network. Health Serv Res 28: 459-478.

15. Burt R (1995) Structural holes: The social structure of competition. Harvard University Press. Paper back edition : 324 .

16. Cary NC (2004) SAS/STAT 9.2 user's Guide introduction to Statistical modeling with SAS/STAT Software. SAS Institute Inc.

17. Borgatti, SP, Everett MG, Freeman LC (2002) Ucinet for Windows: Software for social network analysis. Harvard Analytics Technologies 15: $12-15$.

18. Borgatti SP (2002) NetDraw: graph Visualization Software. Harvard Analytic Technologies.

19. Leone S (2017) Sierra Leone: Mudslides and Floods. The Assessment Capacities Project.

20. World Health Organization (2014) WHO statement on the meeting of the International Health Regulations Emergency Committee regarding the 2014 Ebola outbreak in West Africa.

21. Li J, Duan HJ, Chen HY, Ji YJ, Zhang X, et al. (2016) Age and Ebola viral load correlates with mortality and survival time in 288 Ebola virus disease patients. Int J Infect Dis 42: 34-49.

22. Ansumana R, Jacobsen KH, Sahr F, Idris M, Bangura H, et al. (2015) Ebola in freetown area, sierra leone - A case Study of 581 Patients. N Engl J Med 372: 587-588.

23. Zhang X, Rong Y, Sun L, Liu L, Su H, et al. (2015) Prognostic analysis of patients with Ebola virus disease. PLoS Negl Trop Dis 9: e0004113.

24. Yan T, Mu J, Qin E, Wang Y, Liu L, et al. (2015) Clinical characteristics of 154 patients suspected of having Ebola virus disease in the Ebola holding center of Jui Government Hospital in Sierra Leone during the 2014 Ebola outbreak. Eur J Clin Microbiol Infect Dis 30: 30. 